\section{Bases biológicas e evidências epidemiológicas da contribuição do crescimento fetal e pós-natal na composição corporal: uma revisão}

\section{The biological bases of and epidemiological evidence for the contribution of fetal and postnatal growth to body composition: a review}

Fabiana Cristina Lima da Silva Pastich Gonçalves 1 Rosemary de Jesus Machado Amorim 2 Suzana Maria Ramos Costa 3 Marilia de Carvalho Lima 4

1-4 Universidade Federal de Pernambuco. Av. Prof. Moraes Rego, 1235. Cidade Universitária. Recife, PE, Brasil. CEP: 50.670-901. E-mail: fabiana_pastich@yahoo.com.br

\begin{abstract}
Objectives:to present the biological bases of and epidemiological evidence for fetal and postnatal growth in relation to size and body composition.

Methods: a search of papers published in the last 15 years was carried out in the Lilacs, SciELO, and Medline databases using the key words: growth, fetal growth restriction, low birth weight, catch-up growth, body composition, body mass index and hormones. The studies were selected according to relevance to the evidence to be analyzed.

Results: the articles show the influence of intrauterine growth restriction on the suppression of thermogenesis and hormonal regulation, which in turn interfere with weight gain after birth, and explain how both processes, fetal growth restriction and rapid postnatal weight gain influence body measurements in later life, with consequences that might affect generations.

Conclusions: fetal growth influences the pattern of postnatal growth due to several factors related to hormone regulation, but there is still a gap on the contribution of the combination of fetal growth and postnatal size to body composition in later life.
\end{abstract}

Key words Growth, Fetal growth, Low birth weight, Body composition, Hormones

\section{Resumo}

Objetivos: apresentar as bases biológicas e evidências epidemiológicas do crescimento fetal e pós-natal relacionadas ao tamanho e composição corporal.

Métodos: a busca de artigos publicados nos últimos 15 anos foi realizada nas bases de dados Lilacs, SciELO, Medline através dos descritores: crescimento, restrição do crescimento fetal, baixo peso ao nascer, aceleração compensatória do crescimento, composição corporal, indice de massa corporal e hormônios. Os estudos foram selecionados de acordo com a pertinência às evidências a serem analisadas.

Resultados: os artigos apontam para a influência da restrição do crescimento intraútero na supressão da termogênese e regulação hormonal, que por sua vez interferem no ganho de peso após o nascimento, $e$ explicam como ambos os processos, restrição do crescimento fetal e rápido ganho de peso pós-natal, influenciam as medidas corporais em fases posteriores da vida, com consequências que poderão afetar gerações.

Conclusões: o crescimento fetal influencia o padrão de crescimento pós-natal devido a diversos fatores relacionados à regulação hormonal, porém existe ainda uma lacuna sobre a contribuição da somação entre crescimento fetal e pós-natal no tamanho e composição corporal em fases posteriores da vida.

Palavras-chave Crescimento, Crescimento fetal, Baixo peso ao nascer, Composição corporal, Hormônios 


\section{Introdução}

Os fatores ambientais perinatais parecem ser determinantes das condições de saúde e doença em idades posteriores. Dentro do útero materno, sob a influência da interação entre os genes e o ambiente, o feto sofre mudanças orgânicas capazes de induzir alterações por todo o ciclo da vida, e estas alterações, quando associadas aos fatores ambientais e genéticos após o nascimento, desencadearão novas transformações no organismo. ${ }^{1}$ Baseada em estudos epidemiológicos e experimentais surgiu a hipótese da "origem desenvolvimentista da saúde e da doença”. Nesta, sugere-se que o desenvolvimento de doenças ou o próprio processo de saúde no futuro são consequências da interação entre fatores pré e pós-natais, o que dá uma ideia de sequência e continuidade da resposta genética às variações ambientais ocorridas durante o ciclo vital, e que serão passados de geração em geração. ${ }^{2}$

Sabe-se que a ocorrência de distúrbios do crescimento em qualquer fase da vida e, principalmente, em seu início, pode causar efeitos deletérios à saúde, o que torna a investigação das suas causas um fator importante para a prevenção dos agravos futuros. A proposta desta revisão é discutir as repercussões do crescimento intraútero e do rápido ganho ponderal pós-natal, nas modificações estruturais do organismo, entre elas, o aumento de peso secundário ao acúmulo de gordura corporal observado na infância, adolescência e na idade adulta.

\section{Programação fetal entre gerações}

A contribuição materna na geração da prole começa tão precocemente quanto o próprio processo da sua gestação, pois os folículos primordiais são originados ainda na primeira metade da vida fetal, quando então ficam quiescentes até a puberdade. ${ }^{3} \mathrm{~A}$ influência das informações ambientais, especialmente as nutricionais, são passadas pela avó para a mãe, que por sua vez dará sua contribuição durante a maturação do oócito, ou seja, ainda numa fase préconcepcional. Estas mensagens serão passadas para o feto que está em formação juntamente com aquelas recebidas nos estágios iniciais da gestação. Este fato é evidenciado, ao se constatar que o peso ao nascer da prole frequentemente se relaciona com o peso materno ao nascimento. ${ }^{4}$ A ocorrência deste fenômeno está de acordo com a ausência de grandes mudanças de uma geração para outra, já que informações da avó são recebidas pela neta.

A nutrição materna durante a maturação do oócito é relevante para o feto em formação, infor- mando-o sobre a disponibilidade de nutrientes durante o seu desenvolvimento e, no período de préimplantação, serão as reservas do próprio óvulo que irão suprir as necessidades energéticas para a multiplicação celular. ${ }^{5}$ Todas as instruções são comunicadas ao novo ser muito precocemente, a fim de que sejam ativados mecanismos compensatórios para mantê-lo vivo caso haja restrição das fontes de alimento. Em resposta, este novo ser será "econômico", reservando sempre um pouco de energia.

Durante o estágio de pré-implantação o embrião é banhado por uma secreção uterina nutritiva. ${ }^{5}$ Após a implantação, o embrião é suprido pela passagem placentária de aminoácidos e glicose que são seus substratos para formação tecidual e produção de energia durante o primeiro trimestre. Neste momento, começa na mãe a resistência à insulina secundária às mudanças hormonais próprias da gestação. A glicemia materna aumenta progressivamente para favorecer o transporte ativo de proteínas e para proporcionar mais glicose como fonte de energia ao cérebro em desenvolvimento.

No final da gestação, as necessidades nutricionais fetais aumentam e a gestante recorre aos estoques de tecido adiposo depositados no primeiro trimestre. Quando a reserva de gordura corporal é utilizada como fonte de energia, os ácidos graxos livres e o glicerol são liberados na corrente sanguínea. Os ácidos graxos serão utilizados como fonte de energia pela mãe e a cetona, resultante do seu metabolismo, será aproveitada pelo cérebro fetal, assim como o glicerol será aproveitado na gliconeogênese para o fornecimento de novos substratos energéticos. 6

Esses dados acerca do metabolismo maternofetal em que se observa resistência à insulina e hiperglicemia materna, bem como o aproveitamento de nutrientes para o favorecimento do crescimento do fetal sugerem que os fetos programados para nascerem pequenos, mas que por algum motivo tiveram um rápido ganho de peso no último trimestre da gestação, podem apresentar-se adequados ou mesmo grandes para idade gestacional ao nascer e com a composição corporal mais rica em tecido adiposo. Isto pode explicar a relação em forma de $\mathrm{U}$ da associação entre o peso ao nascer e o risco de apresentar a obesidade, visto que recém-nascidos pequenos e grandes para a idade gestacional, ou seja, os extremos da curva de peso para a idade gestacional, têm maior risco posterior de apresentar obesidade. 7

Por isso, acredita-se que o feto cuja mãe sofreu restrição do crescimento intraútero obtém instruções 
de ser "econômico", crescer lentamente e ter menor tecido muscular para gastar menos energia, mas se a mãe tiver um maior aporte energético e nutricional desde a maturação do oócito e começo da gestação, este novo ser poderá ter um maior crescimento corporal durante o período fetal. No entanto, ao nascer terá maior massa gordurosa e maior proporção de tecido adiposo em relação ao tecido magro o que poderá predispô-lo à síndrome metabólica independente do seu peso ao nascimento.

Portanto, aventa-se a possibilidade da ocorrência de um catch up intraútero em fetos que, através da herança genética e epigenética de seus antecedentes, receberam a informação de restringir o crescimento prevendo uma escassez das fontes nutritivas, mas que foram supridos de suas necessidades energéticas, e com isso, apresentarem uma desproporção entre tecido magro e gordo corporal ao nascer, com maior proporção de tecido adiposo.

Porém, o feto que sofre restrição de suas necessidades energéticas, responde ao sinal de má nutrição com a adaptação do organismo, alterando o metabolismo e reduzindo o tamanho do corpo. A cronificação desta condição provoca atrofia na formação muscular que ocasiona uma menor proporção massa magra corporal. ${ }^{8}$ Em curto prazo, uma das consequências da restrição do crescimento fetal é o menor peso ao nascer, 9 que representa um menor tamanho dos compartimentos de tecidos corporais.

O recém-nascido que sofreu restrição de seu crescimento dentro do útero materno tentará restabelecer todo o processo de crescimento logo após o nascimento, assim que estiverem normalizados os cuidados neonatais e pós-natais. Assim, constitui-se a aceleração compensatória do crescimento, que pode provocar graves efeitos em longo prazo, dentre estes a alteração da composição corporal, quando se observa uma desproporção entre massa magra e tecido adiposo, ${ }^{8}$ resistência à insulina, 10 síndrome metabólica e as doenças crônicas não transmissíveis. ${ }^{11}$

\section{Crescimento pós-natal}

O crescimento pós-natal é resultante de fatores como hereditariedade, condições socioeconômicas e ambientais 12 e durante a infância difere quanto ao sexo, faixa etária e peso ao nascer. As crianças do sexo masculino apresentam maior peso e comprimento/altura que as do sexo feminino. O período de maior aceleração do desenvolvimento corporal ocorre após o nascimento durante o primeiro ano de vida, especificamente, de zero a seis meses, com uma desaceleração após esta idade. 12 Quanto ao peso ao nascer, alguns autores referem uma relação inversa entre o ganho de peso e o tamanho ao nascimento ${ }^{13,14}$ visto que o incremento médio ponderal e de comprimento nos primeiros meses de vida é maior nos recém-nascidos a termo de menor peso quando comparados aos de maior peso ao nascer, 12,15 com o predomínio do ganho de peso em relação ao crescimento linear. 12

Apesar desta aceleração, dita compensatória da restrição sofrida no período intraútero, ${ }^{13}$ estas crianças permanecem com menores índices antropométricos que àquelas que, aparentemente, não passaram por efeitos deletérios no período intraútero. 12

O rápido aumento do tamanho corporal ocorre quando há o restabelecimento do crescimento após o período de restrição deste, e tem sido reconhecido como um dos mais importantes fatores de risco para complicações metabólicas, devido a sua relação com o armazenamento de gordura corporal, principalmente, a gordura abdominal, 13 sendo considerado como um possível indicador prognóstico da saúde do adulto. 16

\section{Aceleração do ganho de peso}

O ganho acelerado de peso após o nascimento parece associar-se a maiores depósitos de gordura corporal, 17 principalmente, naqueles neonatos que apresentam baixo índice ponderal mesmo tendo nascido com o peso adequado para a idade gestacional.18 Quando este ganho ultrapassa uma determinada faixa do percentil, acima de 0,67 do desviopadrão na curva de crescimento, é considerado um ganho compensatório clinicamente significante. 13

$\mathrm{O}$ fato de crianças nascidas a termo pequenas para a idade gestacional, apresentarem aceleração compensatória no ganho ponderal pós-natal e as grandes para a idade gestacional demonstrarem menor ritmo de crescimento, 13 corrobora com o pressuposto de que o risco para um ganho excessivo de gordura corporal pode derivar da interação entre as consequências da restrição do crescimento intraútero e os fatores genéticos e ambientais pósnatais. ${ }^{19}$ A seguir, são apresentados alguns mecanismos envolvidos no favorecimento do aumento de peso e no acúmulo de tecido adiposo após o nascimento, decorrentes ou não da evidente restrição do crescimento fetal.

\section{Insulina, músculo esquelético e supressão} da termogênese

A insulina é um hormônio anabólico secretado pelas células $\beta$ das ilhotas pancreáticas em resposta 
ao aumento dos níveis circulantes de glicose após as refeições, ${ }^{20}$ sendo um dos principais fatores de crescimento tanto intra quanto extraútero. Entre suas funções a principal é a captação da glicose sanguínea circulante para armazenamento deste substrato em forma de energia.

O músculo esquelético é responsável por cerca de $80 \%$ da captação de glicose em resposta a ação da insulina, além de ser um importante tecido para a produção de energia e controle da deposição de gordura. Observa-se que crianças que sofreram restrição do crescimento intraútero têm menor proporção de tecido muscular $8,21,22$ o que diminui a capacidade de captação e utilização da glicose. Estas deficiências no mecanismo de captação e utilização da glicose causam a elevação da glicemia e, paradoxalmente, em resposta, o organismo aumenta a produção hepática para o suprimento de energia, o que favorece ainda mais a elevação da quantidade de glicose sanguínea circulante resultando em formação de gordura. 23

A ação e a sinalização de insulina no músculo esquelético parecem representar o mecanismo que explica a associação entre a resistência à insulina e a aceleração do ganho de peso. Vaag et al.,23 observaram, por meio de biópsia muscular realizada em adultos nascidos a termo com peso adequado para a idade gestacional e com baixo peso, que existe diferença na quantidade de proteínas responsáveis pelo transporte muscular de glicose entre estes grupos, com uma menor expressão de proteína sinalizadora de glicose nos nascidos com baixo peso. Outro estudo, também conduzido com a realização de biópsia de músculo esquelético de adultos que nasceram com peso abaixo de $3000 \mathrm{~g}$, mostrou redução na expressão de algumas proteínas sinalizadoras de insulina associando-se à resistência insulínica quando comparados com os que apresentaram maior peso ao nascimento. ${ }^{24}$ Este fato favorece a retroalimentação/controle negativo causado por uma menor transmissão intracelular do sinal da insulina no músculo, o que resulta em um estado de hiperinsulinemia.

A presença de insulina no sangue inibe a gliconeogênese e a glicogenólise, e estimula o acúmulo de glicogênio (reserva energética) e a síntese de glicogênio no fígado e no músculo. ${ }^{20}$ Porém, a redução de proteínas transportadoras de glicose e sinalizadoras de insulina no músculo esquelético, permite a suposição que, estes indivíduos apresentam ao mesmo tempo um estado de hiperglicemia e resistência insulínica o que favorece a lipogênese.

Jensen et al.,25 investigaram a ação da glicose intra e extracelular entre adultos que nasceram a termo pequenos para idade gestacional (peso abaixo do percentil 10) e com peso adequado para a idade gestacional (peso acima do percentil 50), observaram reduzida secreção de insulina naqueles que nasceram de baixo peso, o que pode levar à intolerância a glicose, favorecer a lipogênese e consequentemente o acúmulo de gordura e desenvolvimento da obesidade. Sugere-se também que a resistência insulínica estaria relacionada à desproporção entre a quantidade de gordura corporal em relação à massa muscular. ${ }^{26}$

A aceleração do ganho de peso por si é caracterizada por resistência à insulina e hiperinsulinemia. Esta pode levar ao estado de hiperfagia que suprime a produção de calor em alguns tecidos para que haja a formação de adipócitos. A diminuição da produção de calor acarreta acúmulo de gordura corporal e com isso, a distribuição da gordura centralizada e elevação do triglicerídeo intramiocelular e da concentração de lipídio circulante, aumentando a propensão à obesidade caracterizada nem sempre pelo aumento excessivo do peso corporal, mas pela deposição de tecido adiposo visceral e muscular. A lipogênese emite um sinal inibitório para o músculo esquelético que suprime a produção de calor (termogênese) e que redireciona mais glicose para a síntese de gordura. A própria regulação hormonal do estoque dos adipócitos durante a aceleração do ganho de peso, pode levar a resistência à insulina. 27

Como o número de receptores para a oxidação de glicose se encontra reduzido no músculo esquelético devido à privação sofrida por este tecido durante o seu desenvolvimento, a captação, transformação e armazenamento de glicose em forma de glicogênio diminuirão, permitindo que altos níveis de glicose permaneçam circulantes. ${ }^{28}$

\section{Grelina e hiperfagia}

A grelina é um hormônio orexígeno produzido principalmente no estômago e tem uma importante função na tentativa de manter a homeostase de energia através do estímulo à ingestão alimentar. ${ }^{29}$ Além do envolvimento com a manutenção do balanço energético e de sofrer influência dependente da ingestão de alimentos, este hormônio parece ser afetado pela depleção do estado nutricional no período fetal. 30

No cordão umbilical de crianças nascidas pequenas para a idade gestacional, encontra-se uma maior concentração de grelina quando comparadas à concentração deste hormônio encontrada naquelas nascidas com peso adequado. $30 \mathrm{O}$ mesmo pode ser observado ao comparar os níveis séricos de grelina nos recém-nascidos pequenos para a idade gesta- 
cional comparados com os adequados e grandes para a idade gestacional. 31

Em um estudo experimental, Desai et al.,32 observaram um nível elevado de grelina na prole de ratas que foram submetidas à restrição alimentar durante a gestação. Esta prole que sofreu desnutrição intraútero, ao nascer apresentou hiperfagia e permaneceu com o ganho de peso acelerado mesmo após a recuperação do crescimento, o que resultou em aumento de peso e gordura corporal comparado aos ratos nascidos do grupo controle.

Esta associação entre o nível de grelina e o ganho de peso também é relatada em humanos. Aceleração compensatória do crescimento pós-natal pode estar relacionada à variação do nível de grelina circulante, o que reflete o aumento do apetite em crianças nascidas pequenas para idade gestacional. Iñiguez et al., 33 encontraram que a taxa de grelina após a introdução de glicose intravenosa foi positivamente correlacionada com o catch up em peso e com o peso corporal, aos 12 meses, em crianças que sofreram restrição do crescimento intraútero comparadas àquelas nascidas pequenas para idade gestacional, mas que não apresentaram aceleração compensatória do crescimento.

Portanto, o aumento dos níveis de grelina circulantes que, segundo Farquhar et al.,31 teria uma função de adaptação fisiológica da restrição do crescimento fetal, pode contribuir com a aceleração compensatória do crescimento pós-natal, e com isso, com o aumento de gordura corporal.

\section{Balanço energético positivo e inadequação}

\section{alimentar}

Para que haja o crescimento tecidual é necessário um balanço energético positivo, que pode ocorrer por diminuição da taxa metabólica basal, reduzindo a energia despendida, ou por acréscimo no consumo energético, ou ambos. 15

$\mathrm{O}$ alimento oferecido em quantidade e qualidade inadequada para o recém-nascido altera o balanço energético do organismo, que apesar da necessidade aumentada de calorias por se tratar de um período de construção tecidual, sofrerá alterações muito rápidas em sua composição corporal, 15 aumentando demasiadamente o número de células adiposas podendo causar um desequilíbrio na proporção de tecidos corporais, o que influenciará no processo de captação e utilização de energia. ${ }^{27}$

Após um período restritivo de substratos energéticos, com a normalização e estabilização dos cuidados e da alimentação, o nível de glicose circulante no sangue se eleva na tentativa de preservar e armazenar energia para a reconstrução dos tecidos corporais. O estado de realimentação nestas condições já caracteriza um efeito metabólico semelhante ao estado de hiperfagia, quando comparado à privação antecedente, o que leva a uma maior liberação de insulina para a captação da glicose circulante 27 e isso favorecerá o rápido aumento dos adipócitos.

É indiscutível a influência da alimentação no estado de saúde. O consumo de uma dieta desequilibrada em nutrientes na infância parece interferir no ganho de peso e consequentemente no risco de obesidade no futuro. A alta ingestão energética nos primeiros meses de vida está associada ao rápido ganho de peso e ao risco de obesidade na infância. ${ }^{15}$ Um acompanhamento da ingestão alimentar de crianças demonstrou que, quando estas são alimentadas com fórmula láctea, ingerem mais proteína e cerca de 15 a $20 \%$ mais calorias que àquelas em aleitamento materno e, mesmo após iniciarem o consumo de alimentos sólidos, a diferença na quantidade de calóricas e proteínas ingeridas entre estes grupos se mantêm. Neste mesmo estudo, foi observado que o total de energia ingerida foi positivamente relacionado com o ganho de peso a partir do terceiro mês de vida. As crianças em aleitamento materno apresentaram menor ganho de peso, porém, ganharam mais massa muscular por grama de proteína ingerida comparadas às alimentadas com fórmula láctea, que apresentaram maior ganho de massa gorda. 34

A utilização metabólica de nitrogênio depende do tipo de proteína ingerida. Embora as crianças que consomem fórmulas lácteas apresentem uma maior ingestão de proteínas, a quantidade e a composição protéica do leite materno são mais próximas dos requerimentos nutricionais, por isso, àquelas que são amamentadas demonstram maior eficiência metabólica na utilização de nitrogênio. ${ }^{34}$

Koletzko et al.,35 observaram que crianças que consumiam fórmulas com elevada proporção de proteína, apresentaram maior peso e índice de massa corporal aos seis meses, comparadas às crianças que ingeriam fórmulas de mesma densidade energética e glicídica, e composição semelhante de lipídeos, porém, com menor quantidade de proteína, o que sugere a influência da dieta hiperprotéica no aumento do peso corporal. Há também evidências da associação entre a elevada ingestão protéica durante os períodos de alimentação complementar e início da transição para a dieta da família com elevados índices de massa corporal e gordura corporal em escolares. 36

Indícios do benefício do aleitamento materno são evidentes na prevenção de sobrepeso e obesidade. 
Um maior tempo de exposição ao aleitamento materno exclusivo demonstra um efeito protetor contra o sobrepeso em pré-escolares. Balaban et $a l ., 37$ observaram que a prevalência de sobrepeso foi maior em pré-escolares que mamaram exclusivamente por menos de quatro meses comparadas aos que mamaram por quatro meses ou mais. Kalies et al., 38 verificaram que o prolongamento do tempo de aleitamento materno exclusivo reduz o risco para o elevado ganho de peso até os dois anos e que esta associação foi altamente dose-dependente. Crianças que nunca mamaram ou que mamaram exclusivamente por um mês tiveram uma maior chance de apresentar um elevado ganho de peso do que àquelas que foram amamentadas exclusivamente por seis meses ou mais.

\section{Tecido adiposo, leptina e adiponectina}

Uma das consequências da restrição do crescimento intraútero é a quantidade reduzida de gordura corporal ao nascer. $39 \mathrm{O}$ tecido adiposo não é apenas um sítio passivo de deposição de lipídios, mas um tecido com funções endócrinas com vários efeitos metabólicos 40 e age como regulador dos hormônios derivados dos adipócitos ajustando o metabolismo corporal e a homeostase da energia, como no caso da sua ação sobre a liberação de leptina. 39

A quantidade de gordura corporal é relativamente constante mesmo com as variações na ingestão de energia, podendo manter-se por grande período com pouca alteração, porém quando há redução ou privação energética ocorrendo posteriormente a realimentação, o peso corporal é compensado levando ao aumento do tamanho e do número de adipócitos. 40

Apesar da influência que a gordura do corpo apresenta na concentração de leptina, os adipócitos não podem ser vistos como determinantes da concentração e da sensibilidade periférica a este hormônio. Em obesos, são observados altos níveis plasmáticos de leptina e, apesar da grande quantidade de tecido adiposo, apresentam resistência à leptina, o que permite a predominância da insulina na regulação da concentração sérica deste hormônio, favorecendo o aumento da utilização de glicose pelos adipócitos. 40

A leptina é um hormônio anorexígeno derivado dos adipócitos que age na taxa de regulação corporal. ${ }^{41}$ No período neonatal, este hormônio esta associado com o peso e com o índice de massa corporal, apesar disso, no primeiro ano de vida, as crianças que sofreram restrição intraútero apresentam maiores níveis de leptina circulante quando comparadas as nascidas com peso adequado. ${ }^{42} \mathrm{Sua}$ ação na perda de peso não seria apenas no controle do apetite, mas na regulação do estoque de gordura e dissipação do calor pelo músculo esquelético. Apesar da termogênese no músculo esquelético causar um incremento direto e interdependente em $20 \%$ na taxa metabólica basal, experimentos demonstram que em animais submetidos à desnutrição no período intraútero, mesmo restabelecida a ingestão alimentar após o nascimento, estes efeitos na produção de calor e regulação do estoque de gordura corporal seriam inibidos por apresentarem resistência à leptina o que estaria associado aos altos níveis de insulina circulante (hiperinsulinemia). ${ }^{41}$

A má nutrição pré-natal predispõe a alterações metabólicas que, em um ambiente extraútero propício ao ganho de peso, podem influenciar a regulação e a sensibilidade à leptina. A literatura demonstra que animais que sofreram desnutrição intraútero desenvolvem resistência periférica a este hormônio independente do consumo de uma dieta hipercalórica do tipo hiperlipídica ou hiperglicídica, sendo observados altos níveis de triglicerídeos e insulina associados à resistência à leptina. 43

A qualidade da dieta parece influenciar mais no ganho de peso do que o nível de leptina, tendo em vista que a alta ingestão energética baseada em uma dieta hiperlipídica acelera o ganho de peso, pois, mesmo com altos níveis plasmáticos deste hormônio, os animais parecem não apresentar controle na regulação da homeostase de energia, mas demonstram o desenvolvimento da obesidade, aumento da massa gorda corporal e rápido ganho de peso, 43 evidenciando que a regulação dos níveis de adipócitos são influenciadas pelos macronutrientes da dieta.

Este fato leva à hipótese de que crianças que sofreram restrição do crescimento, principalmente, em períodos que deveriam ter rápido aumento tecidual, sejam no período pré- ou pós-natal, desenvolvem resistência à leptina como uma resposta adaptativa, permitindo a perda de eficiência na regulação deste hormônio para que haja incremento no balanço energético, o que poderá também refletir um defeito no tecido adiposo ocasionado pelo sucessivo e rápido aumento de peso. 42

Outro hormônio produzido e secretado pelo tecido adiposo é a adiponectina, que está envolvida na regulação da glicemia e metabolismo de lipídeos. 44 A restrição do crescimento intraútero parece afetar o metabolismo da adiponectina. ${ }^{45}$ No cordão umbilical de crianças que sofreram restrição do crescimento fetal, o nível deste hormônio encontra-se diminuído comparado ao encontrado no cordão umbilical das que apresentam peso adequado ao nascer.46 Além de uma menor quantidade de 
adiponectina em crianças que sofreram depleção intraútero, Cianfarani et al.,47 perceberam níveis ainda mais baixos para aqueles pequenos ao nascer que apresentaram recuperação do crescimento após o nascimento.

A concentração de adiponectina parece variar durante o crescimento pós-natal. Bozzola et al., 48 avaliaram os níveis de leptina e adiponectina nos grupos de crianças com peso ao nascimento $<-2$ desvios-padrão e $>-2$ desvios-padrão para a idade gestacional e observaram que, para ambos os grupos, o nível de adiponectina aumentou no primeiro mês mas, com o aumento da idade, houve uma diminuição progressiva deste hormônio, enquanto que a leptina se manteve maior durante todo o primeiro ano de vida naqueles com maior peso ao nascer. O padrão de isoforma de adiponectina é alterado em crianças pequenas para idade gestacional o que Ibáñez et al.,49 supõem ser uma estratégia adaptativa de sobrevivência preparando-os para a aceleração compensatória do crescimento pósnatal.

\section{Peso ao nascer e medidas corporais}

Apesar do pressuposto de que o peso ao nascer teria uma relação negativa com o ganho de peso e, consequentemente, com as medidas corporais em idades posteriores são observados dados conflitantes para esta associação. Isto possivelmente se deve aos diferentes métodos utilizados para classificar o crescimento fetal através do peso ao nascimento e ao número reduzido de estudos que controlam os fatores de confundimento. As classificações de baixo peso ao nascer (peso menor que $2500 \mathrm{~g}$ ) para os que nascem entre 37 e 42 semanas de gestação12 e pequeno para a idade gestacional (peso menor que percentil 10)18 ou (< -2 desvio-padrão $)^{48}$ são inferidas nos estudos como uma proxy da restrição do crescimento intrauterino.

Há evidências na literatura sobre a associação do peso ao nascer com o estado nutricional, avaliado através do índice de massa corporal, em pré-escolares, ${ }^{50}$ assim como uma forte e independente associação entre crianças com grande peso ao nascer e o sobrepeso e obesidade na infância. 51 Para Reilly et al.,52 um aumento em cada $100 \mathrm{~g}$ do peso ao nascer é um fator de risco relacionado à obesidade na infância apresentando associação independente e linear com uma maior prevalência de obesidade aos sete anos. Contudo, Joglekar et al., 10 não encontraram associação entre o peso ao nascer e o peso na infância, o mesmo sendo observado por Botton et $a l ., 53$ e Singhal et al.,22 em relação ao índice de massa corporal e a massa gorda em adolescentes. Para Amorim et al.,54 o estado nutricional de crianças na idade escolar foi pouco influenciado pelo peso ao nascimento. Neste mesmo grupo de crianças, a composição corporal avaliada por meio da prega cutânea triciptal não se mostrou diferente entre os nascidos a termo com baixo peso e adequado. 55

Para outros autores, a associação positiva entre o peso ao nascer e o estado nutricional em fases posteriores da vida se deve à diferença na composição corporal, demonstrando que o peso ao nascimento tem associação linear com a quantidade de massa magra, 22 assim como, também pode-se observar que a relação entre o peso ao nascer e o índice de massa corporal e circunferência da cintura em jovens diminui quando o peso do recém-nascido é associado à quantidade de massa magra corporal.21 Para Elia et $a l ., 56$ um aumento em um desvio-padrão no peso ao nascimento associou-se à diminuição de 1,95\% de gordura na puberdade, encontrando que os seguimentos corporais, altura, comprimento das pernas e tecidos magros tendem a ser maiores quanto mais pesada for a criança.

A programação da composição corporal intraútero parece explicar a associação positiva entre peso ao nascer e o maior índice de massa corporal na infância e entre o menor peso ao nascer e o maior índice de massa corporal em adultos. A restrição do crescimento fetal programa uma menor proporção de massa muscular, 22 sendo assim, as crianças pequenas para a idade gestacional comparadas às de peso adequado ou grandes, apresentam menor quantidade de massa magra, $8,21,22$ tendo o peso ao nascer uma relação positiva com a massa muscular. Com isso, o maior peso em recém-nascidos reflete o incremento de tecido muscular durante o período fetal. ${ }^{21} \mathrm{Em}$ crianças com baixo peso ao nascer, por estes apresentarem um maior ganho ponderal pós-natal, suscita a possibilidade deste maior incremento ser devido ao aumento de tecido adiposo.

$O$ rápido aumento de peso pós-natal também apresenta uma relação com a proporcionalidade corporal ao nascer visto que, as crianças nascidas com peso adequado apresentam aceleração do crescimento quando estas são mais magras ${ }^{18}$ ou têm baixo índice ponderal ao nascer. ${ }^{13}$

\section{Rápido crescimento ponderal e medidas corporais}

Independente de como é avaliado o ganho ponderal, seja por velocidade de incremento médio de peso por mês de vida ou por ganho de peso, em desviopadrão, para a diferença entre duas idades, o que se 
observa é uma importante associação entre o rápido ganho ponderal com o sobrepeso e com maiores medidas de gordura corporal em idades posteriores.

Dados publicados na última década ressaltam que o estado nutricional de crianças ${ }^{50,57}$ e adultos 11 estão associados ao rápido ganho de peso após o nascimento. Este que tem sido definido como um ganho de peso maior que 0,67 do desvio-padrão entre duas idades, está associado ao maior índice de massa corporal nas idades pré-escolar, ${ }^{13}$ escolar ${ }^{18} \mathrm{e}$ adolescência. ${ }^{58}$ Para Monteiro et al., 59 um ganho maior que 0,67 desvios-padrão entre zero e 20 meses de idade para os índices peso para idade, comprimento para idade e peso para comprimento, apresentou relação com o sobrepeso na adolescência.

O índice de massa corporal na infância apresenta uma associação direta e positiva com o ganho de peso no primeiro mês de vida. $50 \mathrm{Em}$ crianças não amamentadas, observa-se que quanto maior o incremento de peso por mês nos primeiros quatro meses de vida, maior a associação com o sobrepeso na idade escolar. 57 Já em adultos, segundo Stettler et al.,11 após avaliarem o crescimento infantil em sete ocasiões diferentes, o sobrepeso esteve associado ao ganho em $100 \mathrm{~g}$ no peso na primeira semana de vida. Apesar do índice de massa corporal ser pouco sensível para a investigação de sobrepeso e obesidade, quando associado às medidas de gordura corporal, passam a ser bons identificadores destes riscos. Neste caso, a circunferência da cintura se torna um instrumento importante na identificação de gordura corporal. 13 Observa-se que as crianças que apresentam um rápido crescimento pós-natal têm maiores médias de circunferência da cintura na idade pré-escolar,13,58 escolar, 18 adolescência e na vida adulta 16 quando comparadas com as que não apresentaram aceleração no crescimento.

Para Ong et al., 13 um rápido ganho de peso entre o nascimento e os dois anos esteve associado a maiores medidas corporais de distribuição de gordura central, avaliada através da circunferência da cintura, e ao maior percentual de gordura total em crianças aos cinco anos de idade. O mesmo foi observado por Cameron et al., ${ }^{18}$ que encontraram uma associação direta entre a aceleração compensatória do crescimento e uma maior média de circunferência da cintura na idade escolar. Maiores medidas de massa gorda e circunferência da cintura também foram encontradas em adolescentes acompanhados desde o nascimento, que tiveram um aumento ponderal maior que 0,67 desvio-padrão nos primeiros seis meses de vida. ${ }^{14}$

Para Botton et al., 53 após investigarem os períodos de velocidade de crescimento nas idades entre o nascimento e cinco anos, encontraram que a aceleração no ganho de peso entre o nascimento e os três meses esteve correlacionada com uma maior massa gorda e circunferência da cintura na adolescência. O rápido padrão de crescimento nos primeiros meses de vida também está associado ao risco de obesidade aos sete anos de idade, independente do peso ao nascer. 57

O crescimento é o indicador das condições de saúde da criança, e quando adequado durante o período fetal e durante a infância leva ao ganho de peso harmônico com um aumento proporcional entre ossos, músculos e tecido adiposo, 60 mas, quando há aceleração descompensada neste ganho, tende-se a observar um acréscimo no tamanho das medidas corporais que podem acarretar ao indivíduo um maior acúmulo de gordura independente de este ter desenvolvido obesidade. 18

Acredita-se que o peso ao nascer e a aceleração compensatória do ganho ponderal no início da vida influenciem as medidas corporais de forma independente. O peso ao nascer define a quantidade de massa magra e a aceleração compensatória do crescimento pós-natal determina a massa gorda em idades posteriores. A associação entre a menor proporção de tecido muscular, derivada da restrição do crescimento intraútero, e a maior proporção de massa gorda derivada da aceleração compensatória do crescimento, poderá predispor às doenças crônicas não transmissíveis no futuro.

\section{Referências}

1. Eriksson JG, Forsén T, Tuomilehto J, Jaddoe VW, Osmond C, Barker DJ. Effects of size at birth and childhood growth on the insulin resistance syndrome in elderly individuals. Diabetol. 2002; 45: 342-8.

2. Gluckmann PD, Hanson MA. Developmental origins of disease paradigm: a mechanistic and evolutionary perspective. Pediatr Res. 2004; 56: 311-7.

3. Gosden R, Lee B. Portrait of an oocyte: our obscure origin. J Clin Invest. 2010; 120: 973-83.
4. Horta BL, Gigante DP, Osmond C, Barros FC, Victora CG. Intergenerational effect of weight gain in childhood on offspring birth weight. Int J Epidemiol. 2009; 38: 724-32.

5. Purcell SH, Moley KH. Glucose transporters in gametes and preimplantation embryos. Trends Endocrinol Metab. 2009; 20: 483-9.

6. King JC. Maternal obesity, metabolism, and pregnancy outcomes. Annu Rev Nutr. 2006; 26: 271-91. 
7. Rogers I. The influence of birth weight and intrauterine environment on adiposity and fat distribution in later life. Int J Obes. 2003; 27: 755-77.

8. Heidger ML, Overpeck MD, Kuczmarski RJ, McGlynn A, Maurer R, Davis WW. Muscularity and fatness of infants and young children born small-or-large-for-gestacional-age. Pediatrics. 1998; 102: e60.

9. Godofrey KM, Barker DJP. Fetal nutrition and adult disease. Am J Clin Nutr. 2000; 71 (Suppl.): 1344S-52.

10. Joglekar C, Fall CHD, Deshpand VU, Joshi N, Bhalerao A, Solat V, Deokar TM, Chougule SD, Leary SD, Osmond C, Yajnik CS. Newborn size, and childhod growth, and cardiovascular disease risk factors at the age of 6 years; the pune maternal nutrition study. Int J Obes. 2007; 31:1534-44.

11. Stettler N, Stallings VA, Troxel AB, Zhao J, Schinnar R, Nelson SE, Ziegle EE, Strom BL. Weight gain in the first week of life and overweight in adulthood: A cohort study of European American subjects fed infant formula. Circulation. 2005; 111: 1897-903.

12. Eickmann S H, Lima MC, Motta MEFA, Romani SAM, Lira PIC. Crescimento de nascidos a termo com baixo peso e peso adequado nos primeiros anos de vida. Rev Saúde Pública. 2006; 40:1073-81

13. Ong KKL, Ahmed ML, Emmertt PM, Preece MA, Dunger DB. Association between postnatal catch-up growth and obesity in childhood: prospective cohort study. BMJ. 2000; 320: $967-71$.

14. Ekelund U, Ong K, Linné Y, Neovius M, Brage S, Dunge $\mathrm{DB}$, et al. Upward weight percentile crossing in infancy and early childhood independently predicts fat mass in young adults: the Stockholm Weight Development Study (SWEDES). Am J Clin Nutr. 2006; 83: 324-30.

15. Ong KK, Emmett PM, Noble S, Ness A, Dunger DB, ALSPAC Study Team. Dietary energy intake at the age of 4 months predicts postnatal weight gain and childhood body mass index. Pediatrics. 2006; 117: e503-8.

16. Leunissen RWJ, Kerkhof GF, Stijnen T, Hokken-Koelega A. Timing and tempo of first-year rapid growth in relation to cardiovascular and metabolic risk profile in early adulthood. JAMA. 2009; 301: 2234-42.

17. Meas T, Deghmoun S, Argmoogum P, Alberti C, LevyMarchal C. Consequences of being born small for gestational age on body composition: an 8-year follow-up study. J Clin Endocrinol Metab. 2008; 93: 3804-9.

18. Cameron N, Pettifor J, De Wet T, Norris S. The relation of rapid weight gain in infancy to obesity and sekeletal maturity in childhood. Obesity. 2003; 11: 457-60.

19. Levy-Marchal C, Czernichow P. Small for gestational age and the metabolic syndrome:which mechanism is suggested by epidemiological and clinical studies? Horm Res. 2006; 65 (Suppl. 3): 123-30.

20. Carvalheira JBC, Zecchin HG, Saad MJA. Vias de sinalização da insulina. Arq Bras Endocrinol Metab. 2002; 46: 419-25.

21. Kahn HS, Narayan KMV, Williamson DF, Valdez R. Relation of birth weigt to lean and fat thigh tissue in young men. Int J Obes. 2000; 24: 667-72.

22. Singhal A, Wells J, Cole TJ, Fewrell M, Lucas A Programming of lean body mass: a link between birth weight, obesity, and cardiovascular disease? Am J Clin Nutr. 2003; 77: 726-30

23. Vaag A, Jensen CJ, Poulsen P, Brons C, Pilgaad K, Grunnet L, Vielwerth S, Alibegovic A. Metabolic aspects of insulin resistance in individuals born small for gestational age. Hom Res. 2006; 65 (Suppl. 3): 137-43.

24. Ozanne SE, Jensen CB, Tingey KJ, Storgaard H, Madsbad $\mathrm{S}$, Vaag AA. Low birth weight is associated with specific changes in muscle insulin-signaling protein expression. Diabetol. 2005; 48: 547-52.

25. Jensen CB, Storgaaard H, Dela F, Holst JJ, Madsbad S, Vaag AA. Early differential defects of insulin secretion and action in 19-years-old Caucasian how had low birth weight. Diabetes. 2002; 51: 1271-80.

26. Dulloo AG, Jacquet J, Montani JP. Pathways from weight fluctuations to metabolic diseases: focus on maladaptive thermogenesis during catch-up fat. Int J Obes. 2002; 26: S46-57.

27. Dulloo AG. Regulation of fat storage via suppressed thermogenesis: a thrifty phenotype that predisposes individuals with catch-up growth to insulin resistance and obesity. Horm Res. 2006; 65 (Suppl. 3): 90-7.

28. Dulloo AG, Gluber M, Montani JB, Seydoux J, Solinas G Substrate cycling between de novo lipogenesis and lipid oxidation: a thermogenic mechanism against skeletal muscle lipotoxicity and glucolipotoxicity. Int J Obes. 2004; 28: S29-37.

29. Kojima M, Kangawa K. Ghrelin: structure and function. Phys Rev. 2005; 85: 495-522.

30. Önal EE, Cinaz P, Atalay Y, Türkyilmaz C, Bideci A, Aktürk A, Okumus N, Unal S, Koç E, Ergenekon E. Umbilical cord ghrelin concentrations in small- and appropriate-for-gestational age newborn infants: relationship to anthropometric markers. J Endocrinol. 2004; 180: 267-71.

31. Farquhar J, Heiman M, Wong ACK, Wach R, Chessex P, Chanoine JP. Elevated umbilical cord ghrelin concentrations in small for gestational age neonates. J Clin Endocrinol Metab. 2003; 88: 4324-7.

32. Desai M, Gayle D, Babu J, Ross MG. Programmed obesity in intrauterine growth-restricted newborns: modulation by newborn nutrition. Am J Physiol. 2005; 288: 91-6.

33. Iñiguez G, Ong K, Peña V, Avila A, Dunger D, Mericq V. Fasting and post-glucose ghrelin levels in SGA infants: relationships with size and weight gain at one year of age. J Clin Endocrinol Metab. 2002; 87: 5830-3.

34. Heinig MJ, Nommsen LA, Peerson JM, Lonnerdal B, Dewey KG. Energy and protein intakes of breast-fed and formula-fed infants during the first year of life and their association with growth velocity: the Darling Study. Am J Clin Nutr. 1993, 58: 152-61

35. Koletzko B, von Kries R, Monasterolo RC, Subías JE, Scaglioni S, Giovannini M, Beyer J, Demmelmair H, Gruszfeld D, Dobrzanska A, Sengier A, Langhendries JP, Cachera MFR, Grote V, for the European Childhood Obesity Trial Study Group . Lower protein in infant formula is associated with lower weight up to age $2 \mathrm{y}$ : a randomized clinical trial. Am J Clin Nutr. 2009; 89: 1836-45.

36. Gunther ALB, Buyken AE, Kroke A. Protein intake during the period of complementary feeding and early childhood 
and the association with body mass index and percentage body fat at $7 \mathrm{y}$ of age. Am J Clin Nutr. 2007; 85: 1626-33.

37. Balaban G, Silva GAP, Dias MLCM, Dias MCM, Fortaleza GTM, Morotó FMM, Rocha ECV. O aleitamento materno previne o sobrepeso na infância? Rev Bras Saúde Mater Infant. 2004; 4: 263-8.

38. Kalies H, Heinrich J, Borte N, Schaaf B, von Berg A, von Kries R, Wichmann HE, Bolte G, LISA Study Group. The effect of breastfeeding on weight gain in infants: results of a birth cohort study. Eur J Med Res. 2005; 10: 36-42.

39. Briana DD, Malamitsi-Puchner A. Intrauterine growth restriction and adult disease: the role of adipocytokines. Eur J Endocrinol. 2009; 160: 337-47.

40. Havel PJ. Mechanisms regulating leptin production: implications for control of energy balance. Am J Clin Nutr. 1999; 70: 305-6.

41. Solinas G, Summermatter S, Mainieri D, Gluber M, Pirola L, Wymann MP, Rusconi S, Montani JP, Seydoux J, Dulloo AG. The direct effect of leptin on skeletal muscle thermogenesis is mediated by substrate cycling between de novo lipogenesis and lipid oxidation. FEBS letters. 2004; 53944.

42. Jaquet d, Leger J, Tabone MD, Czernichow P, LevyMarchal C. High serum leption concentrations during catch-up growth of children born with intrauterine growth retardation. J Clin Endocrinol Metab. 1999; 84:1949-53.

43. Krechowec SO, Vickers M, Gertler A, Breier BH. Prenatal influences on leptin sensitivity and susceptibility to dietinduced obesity. J Endocrinol. 2006; 189: 355-63.

44. Berg A, Combs TP, Scherer PE. ACRP30/adiponectin: an adipokine regulating glucose and lipid metabolism. Trends Endocrinol Metab. 2002; 13: 84-9.

45. Evagelidou EN, Giapros VI, Challa AS, Kiortsis DN, Tsatsoulis AA, Andronikou SK. Serum adiponectin levels, insulin resistance, and lipid profile in children born small for gestational age are affected by the severity of growth retardation at birth. Eur J Endocrinol. 2007; 156: 271-7.

46. Kyriakakou M, Malamitsi-Puchner A, Militsi H, Boutsikou T, Margeli A, Hassiakos D, Kanaka-Gantenbein C, Papassotiriou I, Mastorakos G. Leptin and adiponectin concentrations in intrauterine growth restricted and appropriate for gestational age fetuses, neonates, and their mothers. Eur J Endocrinol. 2008, 158: 343-8.

47. Cianfarani S, Martinez C, Maiorana A, Scirè G, Spadoni $\mathrm{GL}$, Boemi S. Adiponectin levels are reduced in children born small for gestational age and are inversely related to postnatal catch-up growth. J Clin Endocrinol Metab. 2004; 89: $1346-51$.

48. Bozzola E, Meazza C, Arvigo M, Travaglino P, Pagani S, Stronati M, Gasparoni A, Bianco C, Bozzola M. Role of adiponectin and leptin on body development in infants during the first year of life. Ital J Pediatr. 2010; 36: 1-8.
49. Ibáñez L, Sebastiani G, Lopez-Bermejo A, Díaz M, GómezRoig MD, Zegher F. Gender specificity of body adiposity and circulating adiponectin, visfatin, insulin, and insulin growth factor-i at term birth: relation to prenatal growth. J Clin Endocrinol Metab. 2008; 93: 2774-8.

50. Tanaka T, Matsuzaki A, Kurumaru R, Kinukaua N, Nose Y, Matsumoto T, Hara T. Association between birthweight and body mass index at 3 years. Pediatr Int. 2001; 43: 641-6.

51. Danielzik S, Czerwinski-Mast M, Langnäse K, Dilba B, Müller MJ. Parental overweight, socioeconomic status and high birth weight are the major determinants of overweight and obesity in 5-7 y-old children: baseline data of the Kiel Obesity Prevention Study (KOPS). Int J Obes. 2004; 28: 1494-502.

52. Reilly JJ, Armstrong J, Dorosty AR, Emmett PM, Ness A, Rogers I, Steer C, Sherriff A, for the Avon Longitudinal Study of Parents and Children Study Team. Early life risk factors for obesity in childhood: cohort study. BMJ. 2005; $1-7$.

53. Botton J, Heude B, Maccario J, Ducimetière P, Charles MA. Postnatal weight and height growth velocities at different ages between birth and 5y and body composition in adolescent boys and girls. Am J Clin Nutr. 2008; 87:1760-8.

54. Amorim RJM, Lima MC, Lira PIC, Emond AM. Does low birthweight influence the nutritional status of children at school age? A cohort study in northeast Brazil. Matern Child Nutr. 2011; 7: 295-306.

55. Lima MC, Dantas HF, Amorim RJM, Lira PIC. Does fetal growth restriction influence body composition at school age? J Pediatr (Rio J). 2011; 87: 29-35.

56. Elia M, Betts P, Jackson DM, Mulligan J. Fetal programming of body dimensions and percentage body fat measured in prepubertal children with a 4-component model of body composition, dual-energy X-ray absorptiometry, deuterium dilution, densitometry, and skinfold thicknesses. Am J Clin Nutr. 2007; 86: 618-24

57. Stettler N, Zemel BS, Kumanyaika S, Stalling VA. Infant weight gain and childhood overweight status in a multicenter, cohort study. Pediatrics. 2002; 109; 194-9.

58. Ekelund U, Ong KK, Linné Y, Neovius M, Brage S, Dunger DB, Wareaham NJ, Rössner S. Association of weight gain in infancy and early childhood with metabolic risk in young adults. J Clin Endocrinol Metab. 2006; 92: 98-103.

59. Monteiro POA, Victora CG, Barros FC, Monteiro LMA. Birth size, early childhood growth, and adolescent obesity in a Brazilian birth cohort. Int J Obes. 2003, 27; 1274-82.

60. Leitch I. Growth and health. Int J Epidemiol. 2001; 30: $212-6$

Recebido em 28 de maio de 2012

Versão final apresentada em 18 de junho de 2012

Aprovado em 23 de julho de 2012 